[16] R. Basri, E. Rivlin, and I. Shimshoni, "Visual homing: Surfing on the epipoles," in Proc. IEEE Int. Conf. Comput. Vis., 1998, pp. 863869.

[17] E. Malis, F. Chaumette, and S. Boudet, " 2 1/2 d visual servoing with respect to unknown objects through a new estimation scheme of camera displacement," Int. J. Comput. Vis., vol. 37, no. 1, pp. 79-97, Jun. 2000.

[18] A. Shashua and M. Werman, "Trilinearity of three perspective views and its associate tensor," in Proc. Int. Conf. Comput. Vis., 1995, pp. 920-925.

[19] M. A. Fischler and R. C. Bolles, "Random sample consensus: A paradigm for model fitting with applications to image analysis and automated cartography," Commun. ACM, vol. 24, pp. 381-395, 1981.

[20] G. Cross, A. W. Fitzgibbon, and A. Zisserman, "Parallax geometry of smooth surfaces in multiple views," in Proc. Int. Conf. Comput. Vis., 1999, pp. 323-329.

[21] J. J. Guerrero, R. Martinez-Cantin, and C. Sagüés, "Visual map-less navigation based on homographies," J. Robot. Syst., vol. 10, no. 22, pp. 569$581,2005$.

[22] P. Torr, A. Fitzgibbon, and A. Zisserman, "The problem of degeneracy in structure and motion recovery from uncalibrated image sequences," Int. J. Comput. Vis., vol. 32, no. 1, pp. 27-44, 1999.

[23] A. C. Murillo, J. J. Guerrero, and C. Sagüés, "Robot and landmark localization using scene planes and the 1d trifocal tensor," in Proc. IEEE/RSJ Int. Conf. Intell. Robots Syst., 2006, pp. 2070-2075.

[24] A. C. Murillo, C. Sagüés, J. J. Guerrero, T. Goedemé, T. Tuytelaars, and L. V. Gool, "From omnidirectional images to hierarchical localization," Robot. Auton. Syst., vol. 55, no. 5, pp. 372-382, 2007.

[25] J. A. Castellanos, J. M. Mart'nez, J. Neira, and J. D. Tardós, "Experiments in multisensor mobile robot localization and map building," in Proc. $3 \mathrm{rd}$ IFAC Symp. Intell. Auton. Veh., 1998, pp. 173-178.

\section{From Sensors to Human Spatial Concepts: An Annotated Data Set}

\author{
Zoran Zivkovic, Olaf Booij, Ben Kröse, Elin A. Topp, \\ and Henrik I. Christensen
}

\begin{abstract}
An annotated data set is presented meant to help researchers in developing, evaluating, and comparing various approaches in robotics for building space representations appropriate for communicating with humans. The data consist of omnidirectional images, laser range scans, sonar readings, and robot odometry. A set of base-level human spatial concepts is used to annotate the data.
\end{abstract}

Index Terms-Human-robot interaction, map building, robot space representation.

\section{INTRODUCTION}

Mobile robots are expected to become part of our daily life in the near future. Numerous studies show that people tend to perceive robots

Manuscript received April 4, 2007. This paper was recommended for publication by Associate Editor W. Burgard and Editor H. Arai upon evaluation of the reviewers' comments. This work has been supported in part by the European Commission under Contract EU FP6-002020.

Z. Zivkovic, O. Booij, and B. Kröse are with the University of Amsterdam, 1098SJ Amsterdam, The Netherlands (e-mail: zivkovic@science.uva.nl; obooij@science.uva.nl; krose@science.uva.nl).

E. A. Topp is with the Royal Institute of Technology (KTH), SE-100 44 Stockholm, Sweden (e-mail: topp@csc.kth.se).

H. I. Christensen is with the Royal Institute of Technology, SE-100 44 Stockholm, Sweden, and also with the Georgia Institute of Technology, Atlanta, GA 30332-0160 USA (e-mail: hic@csc.kth.se).

Color versions of one or more of the figures in this paper are available online at http://ieeexplore.ieee.org.

Digital Object Identifier 10.1109/TRO.2008.918046 as social actors and not just tools, and therefore, expect to communicate with them in a natural way [1], [6], [22]. One of the basic skills of the future home robot is goal-directed navigation, including localization, path planning, and path following. This requires an internal model of the environment. Traditionally, mapping and localization have focussed on metric properties and the feature model has typically been point and line based. In general, metric place specification is a poor match to human instructions; it is more natural to use cognitive concepts used by humans when communicating about the space, e.g., rooms. In addition, object type entities, a common human concept related to space, should be included in the robot space representation.

In this sense, the problem is basically a pattern recognition problem. Most pattern recognition approaches use large data sets to learn to recognize concepts. In many application fields (object recognition, speech understanding), annotated databases of sensory data and corresponding labels (concepts) are available [21]. The databases currently available in the robotics community are mainly focused on the geometrical representations [8]. In order to make progress on learning conceptual representations of space, it is essential to have access to appropriate annotated data sets that enable supervised learning and performance benchmarking against ground truth. It would be ideal to have huge data sets from extensive user studies for various situations. However, it is difficult to provide such a general data set and it is realistic to start with some specific scenarios that might be of broad interest. In this brief, we present a data set that is closely related to the so-called "home tour" scenario. This scenario describes a hypothetical situation in which a person receives a new robot and shows the robot how the home looks like. The data set contains sensor readings from some typical sensors used in robotics. Furthermore, we define a set of base-level human spatial concepts and annotate the data. The annotation contains the room where each sensor reading was recorded and the objects visible in the current omnicam image. A flexible annotation tool is provided to allow for other annotations.

In Section II of this brief, we give a short overview of the current research in robotics that address the problem of building spatial representations that are suited for communication with humans. In addition, references to the more widely studied domain of localization and mapping are presented. Some links are also made to the related research activities in the cognitive science and computer vision. In Section III, we describe the structure of the provided data set, the design principles, and some related practical issues. Section IV identifies the type of evaluation schemes that can be performed using the presented data set. The conclusions and our final remarks are listed in Section V.

\section{RELATED WORK}

Most traditional map-building methods in robotics represent geometric properties of the environment, such as occupancy grids or polygonal representations of free space [3]. Another common space representation in robotics is the topological map. A topological map [2], [9], [14] describes the environment as a graph structure with nodes representing distinctive places and edges representing possible transitions.

The traditional maps in robotics are mainly related to the robot navigation task. In order to design space representations appropriate for communicating with humans, extensive user studies and results from cognitive psychology are often considered. In "The intelligent use of space" [11], Kirsh stated that to understand complex (human) models of an environment, we have to observe the interaction of the (human) agent with and within the environment. An example of a robot map representation based on findings from cognitive psychology 


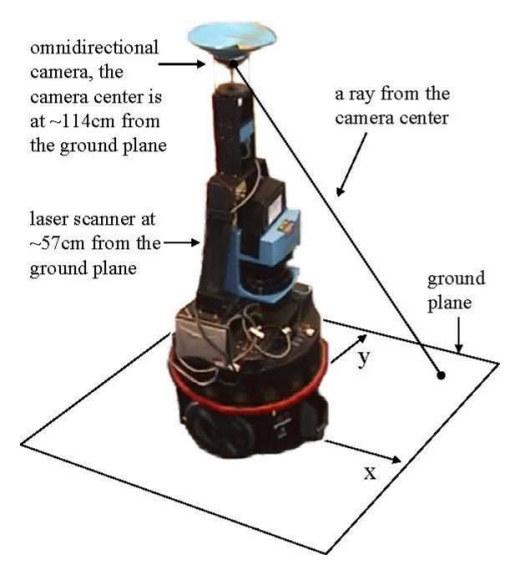

Fig. 1. One of the robots we used for collecting data. The camera axis is approximately aligned with the robot center of rotation. The scanner was mounted so that the origin of the scans had an offset of $\approx 70 \mathrm{~mm}$ in the direction of the $x$-axis of the robot.

research is the "Spatial Semantic Hierarchy" by Kuipers [13], enabling a robot to explore an environment autonomously along the lines of human exploration strategies. Furthermore, a number of researchers report human-robot interaction studies in a guided tour scenario in which a person is guiding a robot [7], [15], [24], [26]. Kruijff et al. [12] consider the issue of clarification dialogues in ambiguous situations of a guided tour. Another important issue is the adaptation of the environment model to the current situation, i.e., personalization. A number of observations from human-robot interaction user studies [7], [24] support the assumption that personal preferences result in quite large variations in what the persons actually presented to the robot during a guided tour.

The reported human-robot interaction studies indicate that hierarchical (or partially hierarchical) models, as could be confirmed with psychological studies [17], become explicit in the interaction of a human with a robot and form a useful base for communication. An indoor environment is typically divided into "delimited regions" (e.g., rooms). The second common concept is that of "object." Particular positions are often described by the relationship to large objects. Our annotation contains objects and rooms in indoor environments.

\section{ANNOTATED DATA SET}

We describe here how we collected the data and the base-level human spatial concepts we used to annotate the data. The data set with annotations is available from: www2.science.uva.nl/sites/cogniron.

\section{A. Data Gathering}

The acquisition of the data set took place in three home environments, see Fig. 2. The mobile robot was driven around by tele-operation to collect the data, see Fig. 1. The following sensors were used.

1) Omnidirectional camera: On average, 7.5 omnidirectional images per second were taken by a camera with a hyperbolic mirror. The $1024 \times 768$ pixel images are in YUV422 color format. The camera is calibrated and images for calibration are available. More information on the omnidirectional vision sensor is presented in the related technical report [28]. The pose of the camera with respect to the robot is known, and a Matlab toolbox is provided for performing basic geometric transformations.

2) Laser scanner: A SICK-laser (LMS-200) was used to record range scans at the front of the robot. The scanner was running in

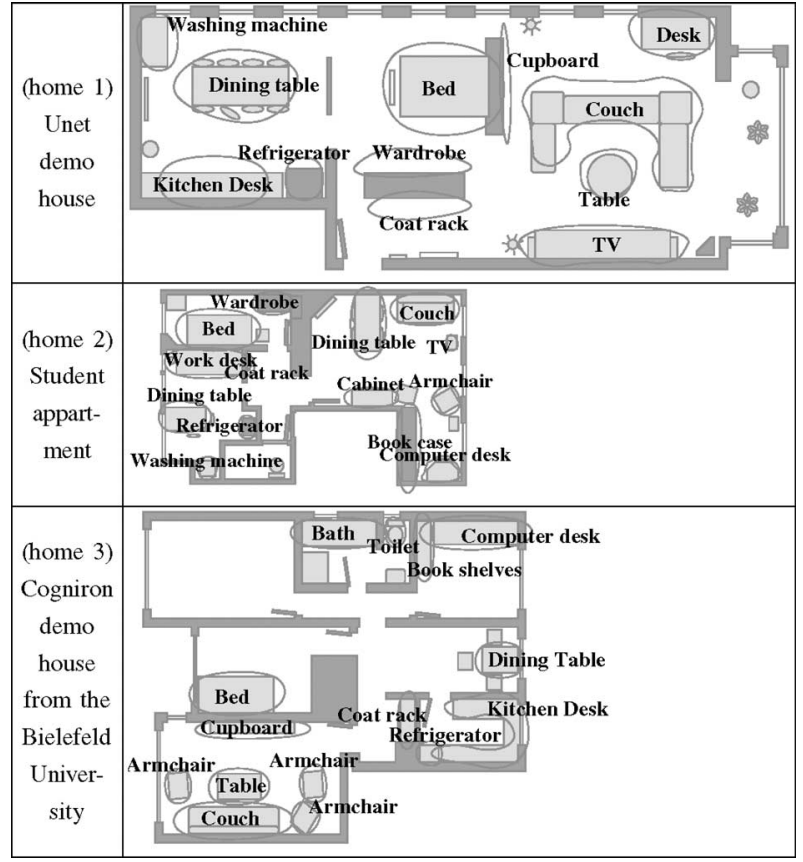

Fig. 2. 2-D maps of the home environments where the data was recorded. The location of the furniture in the drawing is approximate.

millimeter precision, $0.5^{\circ}$ angular resolution over $180^{\circ}$ and had approximately $8 \mathrm{~m}$ maximum range. On average 3.5 scans were conducted per second.

3) Odometry + sonar: On average, 12 odometry measurements per second were taken. Because the robot has solid wheels, the odometry is quite accurate. At the same time, the current values of the 16 ultrasonic sonar sensors were recorded giving a $360^{\circ}$ range scan.

These are the sensors typically used for map building in robotics. An omnidirectional camera was chosen to provide the maximum field of view for navigation. In addition, "low-resolution" rectified images can be resampled from the omnidirectional camera.

The robot was driven through the environment under three different types of conditions.

1) Clean data: We performed two tours by driving the robot at a more or less constant speed, without many people around and with constant lighting.

2) Noisy data: Two tours were performed by driving the robot with people walking around and with more difficult lighting. Furthermore, a number of objects were moved or changed in appearance. These tours were intended to generate more challenging data.

3) Home tour data: Finally, we simulated the so-called home tour scenario where a person is leading the robot around an environment. Again, two tours are performed with two different persons leading the robot. These data are different from the previous runs since there is always a person close to the robot, and the persons tend to stop the robot at certain places in the environment to give explanations about the environment. These data were not meant as a user study, but just to simulate the type of sensory inputs the robot would receive in such a situation.

For our small home environments, each run took just a few minutes. The robot followed a different path in each run. The laser data and the odometry from one of the runs are shown in Fig. 3. For each type of conditions mentioned before, we recorded two runs assuming that typically there will be one training run used to build and learn the 


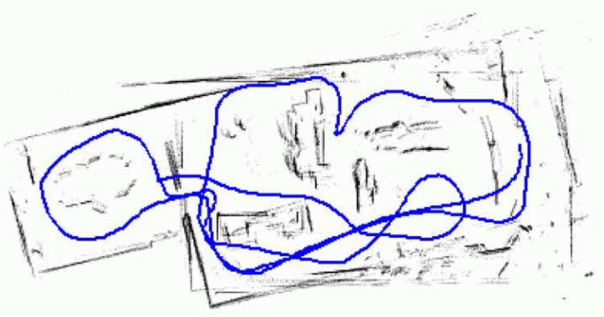

(a)

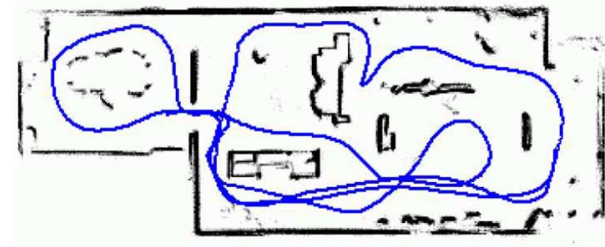

(b)

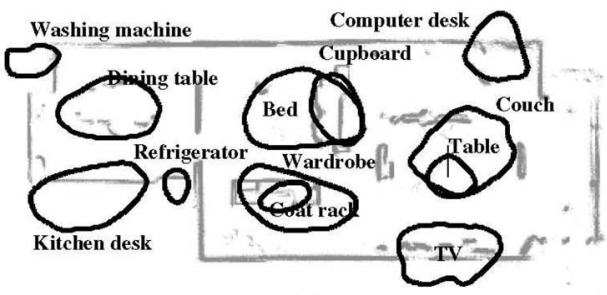

(c)

Fig. 3. Laser scan data and the extracted geometric properties, home 1. (a) Range scans and odometry, (b) Range scans and odometry aligned using SLAM procedure [10], (c) Object visual hull reconstructed from annotations.

environment representation and the second run can be used for evaluation. It is more challenging to use training and testing runs with different conditions.

\section{B. Base Level Concepts and Data Annotation}

We constrain ourselves here to a simple but still rich set of spatial concepts. An indoor environment is typically divided into rooms. The second common concept is that of "object." We selected a number of prominent objects from the environment. The person who annotated the data was supplied with a list of objects, and the task was to segment the objects in the omnidirectional images taken from the robot. The task was also to decide when the robot entered each of the rooms based on the images. The structure of the XML annotation is given in Fig. 4 and Fig. 5 illustrates the annotation.

\section{Toolbox}

In order to allow the researchers a quick start, we provide a Matlab toolbox with a set of functions for accessing the data; the data annotations, and the information about sensor calibration and their positioning on the robot. The provided functions can be divided into following groups.

1) Geometrical: A set of functions that can be used to perform various geometric transformations on the sensory data. For example, a laser scan can be transformed to 3-D world coordinates with respect to the robot, and then, these points can be projected to the omnidirectional camera image.

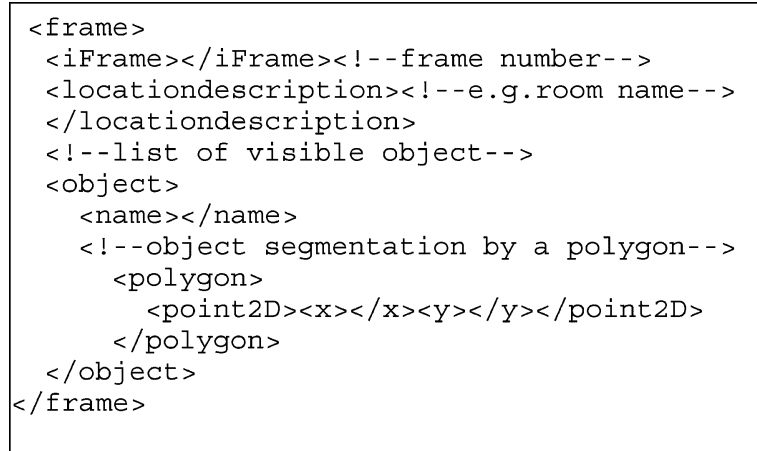

Fig. 4. Basic structure of the XML annotation.

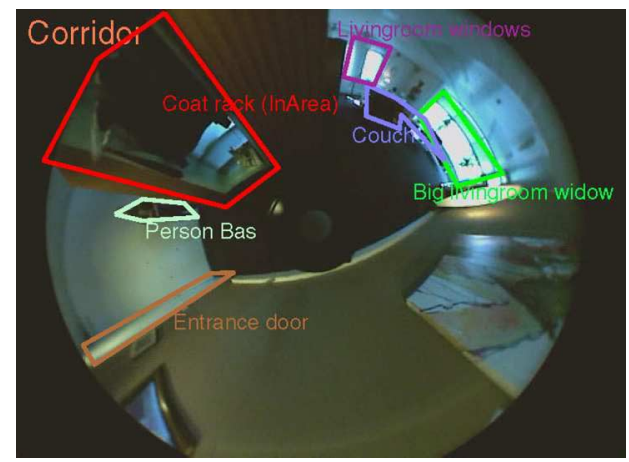

Fig. 5. Example annotated omnidirectional image. The visible objects and persons are segmented using the polygonal lines. The current robot position is denoted as being in the corridor area.

2) Annotation: A data annotation tool is provided visualizing and generating new annotations.

3) Demonstrations: A set of demonstration scripts that illustrate usage of the functions from the toolbox. The images from Fig. 3 are the result of a demo script.

Finally, the provided XML annotation can be transformed to the "Label Me" object database format [21].

\section{Evaluation Methods}

Proper evaluation of a robot space representation that contains human cognitive spatial concepts would consist of extensive user studies where users would interact with the robot in various scenarios. However, it is realistic to start with some specific scenarios and evaluation criteria that might be of broad interest. We concentrate on the so-called "home tour" scenario and identify the following type of evaluation criteria that can be performed using the presented data set.

\section{A. Object and Location Instance Recognition}

One could simulate a realistic learning situation by using the annotation from one run to learn the representation. The annotation from another run from the same environment can be used for the evaluation. The evaluation would consist of testing if the robot can recognize if it revisits a certain location, e.g., room. Recognition of location was reported on the basis of range data [20], [23], or on the basis of visual information [27]. A related topic is the "scene recognition" in image retrieval applications [4]. The robot should also be able to recognize and localize the objects when it observes them again. Object recognition 
is well studied in the computer vision area and robust algorithms are available [16]. While the annotation can be used for evaluating standard algorithms for either recognizing locations or recognizing objects, we encourage algorithms combining these two [25] and also combining the information from different sensors.

\section{B. Object and Location Category Recognition}

The evaluation in the previous section assumes that the robot needs to consider all possible objects and locations in a new environment. On the other hand, from a more advanced home robot, we would expect that it already has knowledge about some concepts, for example, recognizing a TV, a chair, a kitchen, a living room, etc. Evaluating the object (location) category recognition involves learning the representations on one set of home environments and testing it on other previously unseen environments. Currently, the data set contained just three home environments, but this will be extended in the future. The object (location) category recognition algorithms can also be trained on other data sources such as other object databases [18], internet [5], etc, and tested on the presented data set.

\section{Object and Location Geometric Properties}

Traditional map building methods in robotics, i.e., simultaneous localization and mapping (SLAM), concentrate on geometric reconstruction, which is important for robot navigation and/or the task of object grasping. The estimated geometric properties are useful for communicating with humans, e.g., the robot can decide if a room is elongated, one object is larger than the other object, etc. We provide a base line geometric reconstruction as a starting point. First, the data set contains results of a SLAM algorithm based on laser range finder data and line features [10], see Fig. 3(b). Geometric reconstruction of the objects is more difficult. As a base-line approach, we provide a simple algorithm for estimating the 2-D space occupied by the objects. The annotation from the omnidirectional images is back-projected onto a 2-D grid and intersected, the resulting grid cells are the inferred visual hull of the object [19], see Fig. 3(c). We also provide the hand made drawings made by measuring various distances in the environment, see Fig. 2. Note that the focus of data set are the semantic concepts, and the data set is not intended to provide very precise geometric ground truth for evaluating SLAM approaches. Furthermore, home environments are usually not large and do not present a real challenge for current SLAM approaches.

\section{CONCLUSION}

We described, in this brief, an annotated data set, its design principles, and related practical issues. We also propose a set of evaluation criteria. We hope that the annotated data set will be useful for developing, testing, and comparing algorithms for inferring human spatial concepts from sensory data. The data set simulates the home tour scenario currently in three different home environments. In our future work, we aim to extend our data set to more different home environments as well to other types of environments, for example, office environments. Furthermore, additional effort should be made in studying how to compare and evaluate different conceptual representations through extensive users studies. Recently, some initial efforts have been reported in the EU FP6-002020 project Cogniron.

\section{ACKNOWLEDGMENT}

The authors would like to thank the following persons who were involved in making this dataset: A. Doggenaar, B. Terwijn, B. Kröse,
E. Steffens, E. A. Topp, H. Christensen, M. Spaan, O. Booij, R. Boumans, Z. Zivkovic. We would also like to thank UNET for making their space available for the experiments.

\section{REFERENCES}

[1] C. Breazeal, Designing Sociable Robots. Cambridge, MA: MIT Press, 2002

[2] H. Choset and K. Nagatani, "Topological simultaneous localisation and mapping: Towards exact localisation without explicit localisation," IEEE Trans. Robot. Autom., vol. 17, no. 2, pp. 125-137, Apr. 2001.

[3] H. Durrant-Whyte and T. Bailey, "Simultaneous localisation and mapping (SLAM): Part I-The essential algorithms," Robot. Autom. Mag., vol. 13, pp. 108-117, Jun. 2006.

[4] L. Fei-Fei and P. Perona, "A Bayesian hierarchical model for learning natural scene categories," in Proc. IEEE Conf. Comput. Vision Pattern Recog., Jun., 2005, vol. 2, pp. 524-531.

[5] R. Fergus, L. Fei-Fei, P. Perona, and A. Zisserman, "Learning object categories from google's image search," presented at the Int. Conf. Comput. Vision, Beijing, China, 2005.

[6] T. Fong, I. Nourbakhsh, and K. Dautenhahn, "A survey of socially interactive robots," Robots Auton. Syst., vol. 42, pp. 143-166, 2003.

[7] A. Green, H. Hüttenrauch, and E. A. Topp, "Measuring up as an intelligent robot: On the use of high-fidelity simulations for human-robot interaction research," presented at the Workshop Performance Metrics Intell. Syst. (PerMIS), Gaithersburg, MD, 2006.

[8] A. Howard and N. Roy. (2003), "The robotics data set repository (Radish)" [Online]. Available: http://radish.sourceforge.net/

[9] I. Ulrich and I. Nourbakhsh, "Appearance-based place recognition for topological localization," in Proc. IEEE Int. Conf. Robot. Autom., 2000, vol. 2, pp. 1023-1029.

[10] J. Folkesson, P. Jensfelt, and H. I. Christensen, "Vision SLAM in the measurement subspace," in Proc. IEEE Intl. Conf. Robot. Autom., Apr. 18-22, 2005, pp. 30-35.

[11] D. Kirsh, "The intelligent use of space," Artif. Intell., vol. 73, pp. 31-68, 1995.

[12] G.-J. M. Kruijff, H. Zender, P. Jensfelt, and H. I. Christensen, "Clarification dialogues in human-augmented mapping," in Proc. ACM Conf. HumanRobot Interaction, 2006, pp. 282-289.

[13] B. Kuipers, "The spatial semantic hierarchy," Artif. Intell., vol. 119 pp. $191-233,2000$

[14] B. J. Kuipers and Y. T. Byun, "A qualitative approach to robot exploration and map-learning," in Proc. Workshop Spatial Reasoning Multi-Sensor Fusion, 1987, pp. 774-779.

[15] T. Kyriakou, G. Bugmann, and S. Lauria, "Vision-based urban navigation procedures for verbally instructed robots," Robot. Auton. Syst., vol. 51, pp. 69-80, 2005.

[16] D. G. Lowe, "Distinctive image features from scale-invariant keypoints," Intl. J. Comput. Vision, vol. 60, no. 2, pp. 91-110, 2004

[17] T. P. McNamara, "Mental representations of spatial relations," Cognitive Psychol., vol. 18, pp. 87-121, 1986.

[18] J. Ponce, T. Berg, M. Everingham, D. Forsyth, M. Hebert, S. Lazebnik, M. Marszalek, C. Schmid, B. Russell, A. Torralba, C. Williams, J. Zhang, and A. Zisserman, "Dataset issues in object recognition," in Toward Category-Level Object Recognition (Lecture Notes in Computer Science Series), vol. 4170, Berlin, Germany: Springer-Verlag, 2006, pp. 29-48.

[19] M. Potmesil, "Generating octree models of 3D objects from their silhouettes in a sequence of images," Comput. Vision Graph. Image Process., vol. 40, no. 1, pp. 1-29, 1987.

[20] A. Rottmann, O. M. Mozos, C. Stachniss, and W. Burgard, "Semantic place classification of indoor environments with mobile robots using boosting," in Proc. AAAI 2005, pp. 1306-1311.

[21] B. Russell, A. Torralba, K. Murphy, and W. Freeman, "Labelme: A database and web-based tool for image annotation," Intl. J. Computer Vision, 2008, to be published.

[22] B. J. Scholl and P. Tremoulet, "Perceptual causality and animacy," Trends Cognitive Sci., vol. 4, pp. 299-309, 2000.

[23] E. A. Topp and H. I. Christensen, "Topological modelling for human augmented mapping," in Proc. IEEE/RSJ Intl. Conf. Intell. Robots Syst., Oct., 2006, pp. 2257-2263.

[24] E. A. Topp, H. Huettenrauch, H. I. Christensen, and K. S. Eklundh, "Bringing together human and robotic environment representations-A pilot study," in Proc. IEEE/RSJ Intl. Conf. Intell. Robots Syst., 2006, pp. 49464952 
[25] A. Torralba, K. Murphy, W. Freeman, and M. Rubin, "Context-based vision system for place and object recognition," in Proc. Intl. Conf. Comput. Vision, Oct.13-16, 2003, vol. 1, pp. 273-280.

[26] S. Vasudevan, S. Gachter, V. T. Nguyen, and R. Siegwart, "Cognitive maps for mobile robots-An object based approach," Robot. Auton. Syst. J., vol. 55, no. 3, pp. 359-371, May 2007.

[27] Z. Zivkovic, B. Bakker, and B. Kröse, "Hierarchical map building using visual landmarks and geometric constraints," in Proc. IEEE/RSJ Intl. Conf. Intell. Robots Syst., 2005, pp. 7-12.

[28] Z. Zivkovic and O. Booij, "How did we built our hyperbolic mirror omnidirectional camera-Practical issues and basic geometry," Inform. Inst., Univ. Amsterdam Amsterdam, The Netherlands, Tech. Rep. IAS-UVA05-04, 2005.

\section{Development of the Tactile Sensor System of a Human-Interactive Robot "RI-MAN"}

\author{
Toshiharu Mukai, Masaki Onishi, Tadashi Odashima, \\ Shinya Hirano, and Zhiwei Luo
}

\begin{abstract}
Human-interactive robots, such as those used for nursing, which share humans' environments and interact with them, should be covered with soft areal tactile sensors for safety and dexterous manipulation. We report the successful development of the tactile sensor system of our human-interactive robot named RI-MAN, which can lift up a dummy human.
\end{abstract}

Index Terms-Human-interactive robots, tactile sensors, tactile systems.

\section{INTRODUCTION}

With the advent of an aging society, the demand for humaninteractive robots that can help on-site caregivers by playing a part in nursing humans, particularly the elderly, is increasing. Such humaninteractive robots share humans' environments and interact with them, and should be designed on the basis of different criteria from those of conventional industrial robots.

We have developed a robot named RI-MAN [1], [2] (Fig. 1), as a platform for physical human-robot interaction research. Our ultimate goal for RI-MAN is to help nurse elderly people in their daily lives. Having a size similar to a human $(158 \mathrm{~cm}$ in height and $100 \mathrm{~kg}$ in weight), RI-MAN can perform the task of lifting up a dummy human of $16 \mathrm{~kg}$ in its arms. To skillfully perform such tasks that involve physical contact with humans, RI-MAN is equipped with a tactile sensor system.

Tactile sensors have interested many researchers, and various types of tactile sensors have been proposed so far. Many tactile sensors have been developed on the basis of microelectro-mechanical system (MEMS) technology (for example, [3], [4]). They have a high density

Manuscript received July 9, 2007; revised December 11, 2007. This paper was recommended for publication by Associate Editors C. Laschi and H. Arai and Editor H. Arai upon evaluation of the reviewers' comments.

T. Mukai, T. Odashima, S. Hirano, and Z. Luo are with the Biomimetic Control Research Center, RIKEN, Nagoya 463-0003, Japan (e-mail: tosh@bmc.riken.jp; odsm-tds@nifty.com; hirano@bmc.riken.jp; luoz@bmc.riken.jp).

M. Onishi is with the Advanced Industrial Science and Technology (AIST) Tsukuba Central 2, Tsukuba 305-8568, Japan (e-mail: onishi@ni.aist.go.jp)

Color versions of one or more of the figures in this paper are available online at http://ieeexplore.ieee.org.

Digital Object Identifier 10.1109/TRO.2008.917006

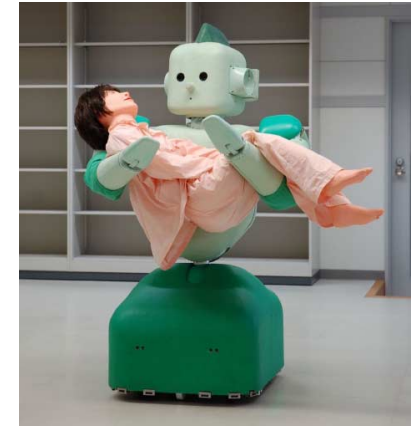

Fig. 1. RI-MAN is a platform for physical human-robot interaction research.

and narrow covering area realized by applying the MEMS technology, and as a result, are not suitable for covering a large area of a robot's surface. Some tactile sensors suitable for use on robot fingers or grippers have also been developed [5]-[7]. Many of them have the ability to the detect tangential stress, and can be used in grasping the force control. Their main target is robot fingers, and consequently, they were not designed to cover a large area. There are also commercially available tactile sensors such as those offered by Tekscan [8], based on the pressure-sensitive ink or rubber and KINOTEX tactile sensors [9], utilizing the change in the intensity of light scattered by the covering urethane foam when deformed. However, they are not sufficiently accurate because of strong hysteresis and creep characteristics.

The idea of covering a large area of a robot's surface with soft tactile skinlike sensors is currently attracting researchers [10]. Tactile sensors for a human-interactive robot that can manipulate objects, and interact and communicate with humans by touch should be soft and compatible with curved robot surfaces. The sensors also need to be able to cover a large area and provide sufficient measurable pressure range, measurement resolution, and spatiotemporal resolution. In addition, the sensors should be stable over a long period, and easily replaceable in case of a malfunction.

To realize skinlike softness, tactile sensors should be composed of or covered with an elastic/viscoelastic material. The effect of such materials on tactile sensors has been discussed in the literature. Fearing and coworkers conducted a mechanical analysis of tactile sensors in cylindrical fingers with a solid core and elastic surface [11]-[14]. Shimojo [15] analyzed and quantified the low-pass filtering effect of an elastic cover. Shinoda et al. [16] proposed a tactile sensor using a tensor cell that detects the stress in the elastic body to determine the structure of the contacting object. More recently, robotic hands with fingers covered with the elastic material have been developed [17]-[19].

Some human-interactive robots for which a large area of their surface is covered with soft tactile sensors have actually been developed [20]-[24]. However, the tactile sensors are not suitable for humaninteractive robots, particularly when a physical labor using a tactile sensation is required. For example, one tactile sensor in [21] has only three values as its output, and another tactile sensor in [21] is gel-type, and cannot be used over a long period because of the evaporation of the contained water. The tactile sensor in [23] has only 56 elements in total. Flexible fabric-based tactile sensors using an electrically conductive fabric have also been proposed for covering a robot [20], but the sensors are binary switches, and are difficult to fabricate. The idea of using the resistance changes of electrically conductive strings in fabric to obtain a strain distribution was also proposed [25], but there are problems in the response accuracy, the stability over time, and the complexity of the electronic acquisition system. To our knowledge, the tactile sensor used by Ohmura et al. [24] has been the most successful 